\title{
Comparação do equilíbrio dinâmico entre praticantes de Brazilian J iu-J itsu com diferentes níveis de experiência
}

CDD. 20.ed. 152.3

796.84

http://dx.doi.org/10.1590/1807-55092015000400535

\author{
Bruno BRASIL* \\ Suzete CHIVIACOWSKY* \\ Fabrício Boscolo DEL VECCHIO* \\ Cristine Lima ALBERTON*
}

\section{Resumo}

0 objetivo do presente estudo foi analisar o equilíbrio dinâmico em praticantes experientes e iniciantes de Brazilian Jiu-Jitsu (BJJ) e grupo controle. A amostra do presente estudo foi composta por 34 participantes, com idades entre 20 e 42 anos, divididos em três grupos: 10 praticantes de BJJ Experientes, 12 praticantes de BJJ Iniciantes e 12 não praticantes de BJJ que compuseram o grupo controle. A tarefa do protocolo consistiu na busca do equilibrio sobre um estabilômetro, com o objetivo de manter a plataforma o mais perto possivel da posição horizontal (ângulo de 0 a $3^{\circ}$ de desvio máximo) durante cada tentativa de 90 segundos. Os resultados demonstram diferenças significativas entre os grupos $[F(2,31)=30,24 ; p<0,001]$. Através dos testes de "post-hoc" foram detectadas diferenças entre o grupo Experientes (46 $\pm 14 \mathrm{~s}$ ) e os outros dois grupos, Iniciantes $(25 \pm 6 s, p<0,001)$ e Grupo Controle $(19 \pm 4 s, p<0,001)$. Todavia, não foram encontradas diferenças significativas entre os Grupo Controle e Iniciantes $(p=0,421)$. Os resultados sugerem que o tempo de prática de BJJ pode influenciar no desempenho do controle do equilibrio.

Palavras-chave: Comportamento motor; Estabilidade; Desempenho motor; Modalidade esportiva de combate.

\section{Introdução}

O Brazilian Jiu-Jitsu (BJJ) é uma modalidade esportiva de combate que objetiva a submissão do adversário, especialmente a partir de técnicas de solo $^{1-2}$. Para que isto ocorra, o atleta busca a projeção do oponente ao solo para, entáo, tentar dominá-lo através de técnicas específicas de estrangulamentos e chaves articulares que se baseiam em posiçóes e alavancas ${ }^{3-4}$. O tempo de combate varia conforme a graduaçáo, de 5 min na faixa branca à 10 min entre faixas pretas; a relação de esforço:pausa é de $6: 1^{5} \mathrm{em}$ lutas oficiais e a maior parte do combate acontece com os oponentes em contato com o solo, sentados, ajoelhados ou deitados ${ }^{6}$.

O BJJ pode ser caracterizado como um desporto de gestos acíclicos ${ }^{2,5}$, pois não apresenta repetitividade nas açôes motoras, nem igualdade na velocidade de execução dos seus movimentos ${ }^{1}$. Neste contexto de prática, observa-se como desafio constante a dificuldade de prever os movimentos dos adversários, a fim de encontrar a melhor forma de responder aos ataques recebidos ${ }^{1,2,7}$.

O domínio do centro de gravidade do corpo humano e da distribuição do peso corporal em açôes dinâmicas pode proporcionar um melhor desempenho em atividades que necessitem bom equilíbrio, como nas lutas ${ }^{8-9}$. Tais parâmetros são difíceis de serem fixados, pois se alteram conforme a movimentação dos diferentes segmentos corporais ${ }^{10}$, inclusive frente às sucessivas e imprevisíveis açôes motoras dos adversários ${ }^{11}$ que provocam instabilidade. Parte deste fenômeno pode ser verificada no estudo de Yosнiтом et al. ${ }^{12}$, que comparou o equilíbrio de judocas em dois diferentes níveis de habilidade: faixa marrom $(8,0 \pm 2,0$ anos de prática) e faixa verde (3,7 $\pm 2,0$ anos de prática). Nele, observou-se que judocas mais experientes apresentaram uma recuperação mais rápida do equilíbrio em comparação aos menos experientes. 
Na luta de BJJ, o atleta se encontra em contato com o adversário durante a maior parte do tempo, e utiliza diferentes sequências de movimentos e manifestaçóes de capacidades motoras para tentar obter sucesso competitivo ${ }^{6}$. Tratando-se das variáveis físicas que contribuem para o êxito na modalidade, destacam-se flexibilidade, força e potência, resistência muscular localizada, condicionamento aeróbio e o equilíbrio ${ }^{2,6,12-14}$. Especificamente quanto ao equilíbrio, nos combates de BJJ o lutador consegue pontos ao projetar o adversário ao solo, ao tirá-lo de cima de si invertendo a posição de luta, movimentos conhecidos como raspagens, e ao conseguir avançar sobre sua defesa, ou seja, passando sua guarda ${ }^{15}$. Em tais movimentos, observa-se que a habilidade em se equilibrar é essencial, porém, substancialmente diferente da posição ortostática ${ }^{8,16}$.

\section{Método}

\section{Delineamento experimental}

Este estudo caracterizou-se como quantitativo comparativo, de caráter transversal e ocorreu em uma cidade do interior do Rio Grande do Sul. Na época do estudo, a cidade contava com sete locais de prática de BJJ que exibiam faixas pretas como responsáveis pelas atividades. Destas, cinco permitiram contato de um dos pesquisadores (BB) com os praticantes. Deste modo, por conveniência foram convidados sujeitos que atendessem aos critérios de inclusão e aceitassem participar voluntariamente do estudo, os quais compuseram a amostra. Após a coleta de dados com os praticantes de BJJ, acadêmicos de Educaçấo Física, com características semelhantes, foram convidados a compor o grupo de nấo praticantes.

Os praticantes de BJJ, não eram competidores e foram alocados em dois grupos: a) 12 indivíduos iniciantes, com graduação entre faixas branca e azul, com no mínimo três meses de prática e, no máximo, três anos; e b) 10 indivíduos experientes, com graduaçáo marrom ou preta, com no mínimo três anos de prática. Os 12 participantes que formaram o grupo controle, não eram praticantes de modalidades esportivas de combate. Para avaliar o equilíbrio dinâmico, uma sessão de coleta de dados foi realizada com cada participante. Os participantes assinaram o Termo de Consentimento Livre e
$\mathrm{Na}$ literatura pesquisada, não foram encontrados estudos sobre o equilíbrio dinâmico em praticantes de BJJ. Considerando a relevância desta capacidade para o sucesso na modalidade e sua importância para essa modalidade esportiva de combate é importante o desenvolvimento de estudos sobre o tema. Portanto, o objetivo do presente estudo foi comparar o equilíbrio dinâmico entre praticantes com diferentes níveis de experiência em BJJ e não praticantes da modalidade. A partir da constatação de que o nível de experiência pode afetar de forma positiva as capacidades físicas e/ou parâmetros bioquímicos dos praticantes $\mathrm{BJJ}^{2-3}$, e considerando os resultados obtidos em pesquisas envolvendo equilíbrio em judocas ${ }^{9,12}$, espera-se que praticantes experientes de BJJ obtenham melhores respostas no controle do equilíbrio, quando comparados a praticantes iniciantes e não praticantes da modalidade.

Esclarecido e o presente estudo foi aprovado pelo Comitê de Ética em Pesquisas com Seres Humanos Local (protocolo n. 21332713.0.0000.5313).

\section{Participante}

A amostra foi composta por 34 participantes, com idade entre 20 e 42 anos. Para cálculo amostral, empregou-se o estudo de Perrin et al. ${ }^{9}$, no qual foi usado estabilômetro para avaliação da capacidade do equilíbrio de judocas de dois níveis competitivos. Assumindo-se diferença mínima entre as médias dos tratamentos de $0,03 \mathrm{~cm}$, desvio padrão do erro de $0,022 \mathrm{~cm}$, três grupos a serem tratados por ANOVA, poder do teste de 0,8 e nível de significância de $5 \%$, foram necessários 12 sujeitos por grupo.

Os dados referentes às características físicas dos três grupos participantes do estudo, Iniciantes, Experientes e Grupo Controle estão descritos na TABELA 1. Valores semelhantes de idade, massa corporal e estatura foram observados entre os três grupos avaliados. Ao comparar os dois grupos de praticantes de BJJ, podem-se observar semelhanças em relação ao tempo de treino semanal, mas diferenças significativas no tempo total de prática entre o grupo Experientes e Iniciantes (TABELA 1).

Foram excluídos da amostra indivíduos que apresentaram problemas vestibulares ou lesões de membros inferiores nos últimos seis meses. 
TABELA 1 - Variáveis de caracterização da amostra entre grupo de praticantes experientes (EX) e iniciantes de Brazilian Jiu-J itsu (IN) e grupo controle (GC).

\begin{tabular}{lcccc}
\hline Variáveis & EX & IN & GC & p-valor \\
& Média \pm DP & Média \pm DP & Média \pm DP & p \\
\hline Idade (anos) & $29 \pm 3$ & $28,3 \pm 5,4$ & $26,4 \pm 6$ & 0,46 \\
Massa corporal (kg) & $80 \pm 8,1$ & $88,6 \pm 11,2$ & $84,6 \pm 8,9$ & 0,12 \\
Estatura (cm) & $174,2 \pm 4$ & $179,3 \pm 5,6$ & $178,7 \pm 7,8$ & 0,13 \\
Tempo de prática de BJJ (anos) & $9,2 \pm 3,1$ & $1,5 \pm 1$ & & $<0,001$ \\
Tempo semanal de treino (horas) & $10,2 \pm 8,1$ & $7,1 \pm 2,7$ & & 0,24 \\
\hline
\end{tabular}

\section{Procedimentos}

A tarefa do presente estudo consistiu na manutenção do equilíbrio sobre a plataforma de um estabilômetro (Modelo 16030, Lafayete Instrument Co.). $\mathrm{O}$ instrumento era composto por plataforma retangular de madeira, medindo $130 \times 140 \mathrm{~cm}$, com desvio máximo de $18^{\circ}$ para a esquerda e para a direita. Os participantes deveriam permanecer com os pés afastados em locais demarcados na plataforma, joelhos semi-flexionados e mãos relaxadas na lateral do corpo, com o objetivo de manter a plataforma o mais perto possível do plano horizontal por 90 segundos, da mesma forma que no estudo de LewTHWaite e WulF ${ }^{17}$. Como variável dependente, foi utilizado o tempo em equilíbrio da plataforma na posição horizontal, com o desvio máximo de $3^{\circ}$ como critério em qualquer um dos lados da mesma (FIGURA 1).

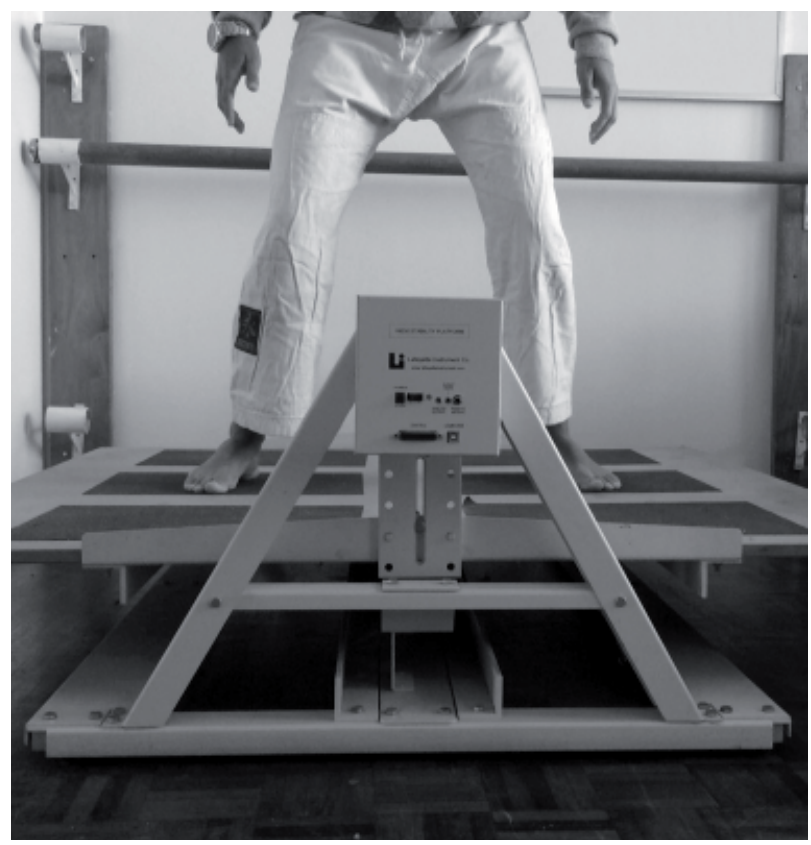

FIGURA 1 - Plataforma de equilíbrio, e posicionamento corporal para o teste de equilíbrio estático.

Cada participante foi conduzido individualmente à sala previamente preparada, onde havia sempre o mesmo experimentador para realizar a coleta, de forma que não houvesse nenhuma interferência do meio externo. Os participantes realizaram duas tentativas, com duração de 90 segundos cada uma, sempre iniciando com o lado esquerdo da plataforma no solo, e com intervalo de descanso de 90 segundos entre elas ${ }^{17}$. Aproximadamente 15 segundos antes do começo de cada tentativa, os participantes foram instruídos a subirem na plataforma com ambos os pés, posicionando-os no centro dos dois marcadores centrais do estabilômetro, mantendo o lado esquerdo apoiado ao solo até que fosse dado o sinal para o início da tarefa. Ao sinal, o sujeito movia a plataforma e a coleta de dados era iniciada. Em cada repetição, os movimentos da plataforma foram monitorados por um potenciômetro e ficaram gravados ao final 
de cada tentativa. A seguir foram obtidas as médias dos escores do tempo em equilíbrio, os quais foram utilizadas para as análises subsequentes.

\section{Análise estatística}

Os dados foram apresentados através de estatística descritiva, média \pm desvio padrão. $O$ teste de Shapiro-Wilk e o teste de Levene foram utilizados para analisar a normalidade dos dados e a homogeneidade das variâncias, respectivamente. Para comparar a variável equilíbrio dinâmico entre os três grupos avaliados (Experientes, Iniciantes e Grupo Controle) foi utilizada a ANOVA "One-way", com "post hoc" de Tukey. Além disso, foi também calculado o tamanho do efeito entre os grupos. Os testes estatísticos foram processados no SPSS versão 19.0, adotando-se um alfa de 5\%.

\section{Resultados}

Tempo em Equilíbrio. A análise dos escores de tempo em equilíbrio demonstraram diferenças significativas entre os grupos $[\mathrm{F}(2,31)=30,24 ; \mathrm{p}$ $<0,001]$. Através dos testes de "post-hoc" foram detectadas diferenças entre o grupo Experientes e os outros dois grupos, Iniciantes $(\mathrm{p}<0,001)$ e Grupo Controle $(p<0,001)$. Não foram encontradas diferenças significativas entre os Grupo Controle e Iniciantes $(\mathrm{p}=0,421)$ (TABELA 2). Ademais, o tamanho do efeito da diferença de desempenho entre Grupo Controle e Iniciantes foi apenas moderado $(E S=1,29)$. Porém, a diferença entre Experientes e Grupo Controle exibiu tamanho de efeito de 6,34, considerado grande, mesma classificação entre Experientes e Iniciantes, com tamanho do efeito de 2,82 .

TABELA 2 - Estatística descritiva (media, desvio padrão e intervalo de confiança) do equilíbrio dinâmico em praticantes de BJJ iniciantes e experientes e um grupo controle.

*indica diferença significativa entre grupo experiente e demais grupos.

\begin{tabular}{lccc}
\hline & $\begin{array}{c}\text { Equilíbrio dinâmico (s) } \\
\text { Média } \pm \text { DP }\end{array}$ & \multicolumn{2}{c}{ Intervalo de confiança 95\% } \\
& Limite inferior & Limite superior \\
\hline Iniciantes & $26 \pm 7$ & 21,3 & 29,7 \\
Experientes & $49 \pm 15^{*}$ & 38,5 & 59,2 \\
Controle & $20 \pm 4$ & 17,2 & 22,5 \\
\hline
\end{tabular}

\section{Discussão}

O principal achado do presente estudo foi que praticantes Experientes obtiveram melhores resultados no equilíbrio dinâmico em comparação a praticantes Iniciantes e não-praticantes. Até o presente momento, não foram encontrados estudos avaliando o equilíbrio em praticantes com diferentes níveis de experiência em BJJ, embora tal capacidade seja fundamental para manutenção do centro de gravidade, e é relevante para praticantes de Brazilian Jiu-Jitsu ${ }^{1,18}$.

Os resultados do presente estudo são similares aos observados no estudo de Yoshiтomi et al. ${ }^{12}$, realizado com o judô, no qual três grupos foram divididos de acordo com faixas de graduação: marrom, verde, sem faixa (controle). Os autores encontraram que os atletas de judô graduados na faixa marrom conseguiram melhor controle do equilíbrio comparado ao grupo controle, e graduados na faixa verde não foram diferentes dos demais grupos, provavelmente por serem praticantes há 3,7 $\pm 2,0$ anos, e não serem considerados iniciantes, mas com experiência intermediária. No presente estudo, iniciantes foram definidos pela graduação nas faixas iniciais, branca e azul, e com três anos de experiência no máximo.

A diferença na capacidade de equilíbrio entre praticantes experientes e não praticantes encontrada no presente estudo pode ser explicada por fatores diversos, como o nível de aptidão física ou grau de aprendizagem motora das habilidades motoras específicas da modalidade. Complementarmente, a diferença entre praticantes experientes e demais envolvidos pode ser devida ao BJJ, assim como o judô, constituir-se de movimentos nos quais os lutadores 
sofrem perturbaçôes inesperadas durante o combate, através de puxões e empurróes realizados por seus adversários $^{5,12}$. Destacam-se, neste sentido, dois pontos relevantes: os vencedores de lutas de BJJ exibem maior frequência de raspagens, projeçóes e passagens de guarda que perdedores; e estas açóes, frequentes nos combates, exibem elevado controle motor e equilíbrio ${ }^{5-6}$. Adicionalmente, tal aprimoramento pode decorrer de diferentes vias, tanto em função da maior diversidade de situaçóes de instabilidade corporal previamente vivenciadas ${ }^{19}$, quanto em função do aprimoramento do controle motor específico e especialização dos hemisférios cerebrais ${ }^{20}$. Deste modo, quando comparados a bailarinas, a imprevisibilidade na origem das açóes motoras da luta é um dos motivos que explica respostas musculares mais rápidas de judocas na manutenção do equilíbrio frente a perturbaçóes do equilíbrio?.

Entretanto, a diferença entre os Experientes e Iniciantes sugere que a quantidade de experiência nas habilidades motoras específicas do BJJ pode afetar a capacidade de equilíbrio, visto que, no presente estudo a quantidade de treino semanal nos últimos três meses foi semelhante entre os grupos. De fato, no estudo de Perrin et al. ${ }^{9}$, quando judocas são comparados a bailarinos em testes de equilíbrio com olhos abertos e olhos fechados, os primeiros obtiveram melhores resultados em testes com olhos fechados. Para os autores, o desempenho dos judocas implica em dominar simultaneamente o equilíbrio dinâmico e estático, dado que os movimentos impostos aos atletas são inesperados ${ }^{9}$. Assim, a prática da modalidade esportiva de combate pode ser importante para a melhora do controle do equilíbrio corporal $^{21-22}$, pois permitiria a experiência em padróes posturais que incluem o controle constante do equilíbrio em função de informações sensoriais nem sempre previsíveis ou claramente disponíveis ${ }^{20,22-23}$. Embora tais inferências decorram de outras modalidades de domínio20,22, cogita-se, considerando os achados do presente estudo, que o treino do BJJ por longo período poderia melhorar, desta forma, a eficiência no equilíbrio estático e dinâmico dos seus praticantes.

Assim, o presente estudo sugere que o equilíbrio dinâmico pode ser variável discriminante entre praticantes de BJJ, além de resistência muscular dinâmica e isométrica de membros superiores indicados por SiLVA et al. ${ }^{24} \mathrm{Na}$ mesma direção, PAILlard et al. ${ }^{19}$ apontam que esportes de combate podem desenvolver capacidades posturais específicas, portanto os movimentos que são praticados em determinado esporte, como por exemplo no Jiu-Jitsu, no qual são exercitadas projeçóes e estabilizaçóes em diversas posiçóes de solo, podem influenciar adaptaçóes posturais induzidas pela aquisição de novas habilidades motoras específicas.

Os resultados do presente estudo sugerem que o nível de experiência de praticantes de BJJ pode influenciar o controle do equilíbrio dinâmico dos mesmos. Participantes experientes apresentam maior controle do equilíbrio postural dinâmico do que praticantes iniciantes ou não praticantes. Como limitações, não foram avaliadas variáveis físicas que poderiam apresentar relaçóes com a prática da modalidade de combate ou relacionadas a aspectos específicos da preparação física de praticantes de BJJ. Portanto, sugere-se que futuros estudos examinem tais variáveis e sua possível influência no controle do equilíbrio entre participantes experientes e iniciantes do desporto. Adicionalmente, sugere-se a avaliação de outras posições específicas do BJJ, e se a prática da modalidade pode modificar parâmetros relacionados ao controle postura dos praticantes.

\section{Abstract}

Comparison of dynamic balance between Brazilian Jiu-Jitsu practitioners with different levels of experience

The aim of the present study was to analyze the dynamic balance in experienced and beginners practitioners of Brazilian Jiu-Jitsu (BJJ) and control group. The sample was composed by 34 participants with ages ranging between 20 and 42 years old, divided into three groups: 10 experienced BJJ practitioners, 12 beginners BJJ practitioners and 12 non-practitioners of BJJ that composed the control group. The task of the protocol consisted on the search for balance over a stabilometer, aiming at keeping the platform as close as possible of the horizontal position during each attempt. The results show significant differences among the groups $[F(2.31)=30,24 ; p<0.001]$. The post-hoc test revealed differences among the experienced BJJ group (46 $\pm 14 \mathrm{~s}$ ) and the other groups (Beginners BJJ: $25 \pm 6 \mathrm{~s}, \mathrm{p}<0.001$; Control group: $19 \pm 4 s, p<0.001)$. However, no significant differences were found between control 
and beginners BJJ groups $(p=0.421)$. The results suggest that the time of BJJ practice may influence on the balance control performance.

KEY WORDS: Motor behavior; Stability; Sensory-motor performance; Sport combat.

\section{Referências}

1. Jones NB, Ledford E. Strength and conditioning for Brazilian Jiu-jitsu. Strength Cond J. 2012;34:60-9.

2. Coswig VS, Neves AHS, Del Vecchio FB. Effects of duration practice in biochemical, hormonal and hematological parameters of people who practice Brazilian jiu-jitsu. Rev Andal Med Deporte. 2013;6:15-21.

3. Coswig VS, Neves AHS, Del Vecchio FB. Biochemical, hormonal and hematological responses to Brazilian jiu-jitsu matches. R Bras Cienc Mov. 2013;21:19-30.

4. Moreira A, Franchini E, Freitas CG, et al. Salivary cortisol and immunoglobulin: a responses to simulated and official Jiu-Jitsu matches. J Strength Cond Res. 2012;26:2185-91.

5. Andreato LV, Franchini E, Moraes SMF, et al. Physiological and technical-tactical analysis in Brazilian jiu-jitsu competition. Asian J Sports Med. 2013;4:137-43.

6. Del Vecchio FB, Bianchi S, Hirata SM, et al. Análise morfo-funcional de praticantes de Brazilian jiu-jitsu e estudo da temporalidade e da quantificação das açóes motoras na modalidade. Mov Percepc. 2007;7:263-81.

7. Andreato LV, Moraes SMF, Esteves JVDC et al. Physiological responses and rate of perceived exertion in Brazilian jiu-jitsu athletes. Kinesiology. 2012;44:173-81.

8. Paillard T, Costes-Salon C, Lafont C, et al. Are there differences in postural regulation according to the level of competition in judoists? Br J Sports Med. 2002;36:304-5.

9. Perrin P, Deviterne D, Hugel F, et al. Judo, better than dance, develops sensorimotor adaptabilities involved in balance control. Gait Posture. 2002;15:187-94.

10. Hall SJ. Biomecânica básica. Rio de Janeiro: Guanabara Koogan; 1993.

11. Imamura RT, Iteya M, Hreliac A, et al. A kinematic comparison of the judo throw Harai-goshi during competitive and non-competitive conditions. J Sports Sci Med. 2007;6:15-22.

12. Yoshitomi SK, Tanaka $\mathrm{C}$, Duarte $\mathrm{M}$, et al. Respostas posturais à perturbação externa inesperada em judocas de diferentes níveis de habilidade. Rev Bras Med Esporte. 2006;12:159-63.

13. Costa EC, Santos CM, Prestes J, et al. Efeito agudo do alongamento estático no desempenho de força de atletas de jiu-jitsu no supino horizontal. Fit Perform J. 2009;8:212-7.

14. Andreato LV. Bases para prescriçáo do treinamento desportivo aplicado ao Brazilian jiu-jitsu. Conexóes. 2010;8:174-86.

15. Paiva L. Pronto pra guerra: preparação física específica para luta \& superação. Manaus: OMP; 2009.

16. Perrot C, Mur JM, Mainard D, et al. Influence of trauma induced by judo practice on postural control. Scand J Med Sci Sports. 2000;10:292-7.

17. Lewthwaite R, Wulf G. Social-comparative feedback affects motor skill learning. Q J Exp Psychol. 2010;63:1-12.

18. Schmidt RA, Wrisberg CA. Aprendizagem e performance motora. 4a ed. Porto Alegre: Artmed; 2010.

19. Paillard T, Montoya R, Dupui P. Postural adaptations specific to preferred throwing techniques practiced by competition-level judoists. J Electromyogr Kinesiol. 2007;17:241-4.

20. Mikheev M, Mohr C, Afanasiev S, et al. Motor control and cerebral hemispheric specialization in highly qualified judo wrestlers. Neuropsychologia. 2002;40:1209-19.

21. Chung P, Ng G. Taekwondo training improves the neuromotor excitability and reaction of large and small muscles. Phys Ther Sport. 2012;13:163-9.

22. Groen BE, Smulders E, Kam D, et al. Martial arts fall training to prevent hip fractures in the elderly. Osteoporos Int. 2010;21:215-21.

23. Leong HT, Fu SN, Ng GY, et al. Low-level Taekwondo practitioners have better somatosensory organisation in standing balance than sedentary people. Eur J Appl Physiol. 2011;111:1787-93.

24. Silva BVC, Junior MM, Simim MAM, et al. Reliability in kimono grip strength tests and comparasion between elite and non-elite Brazilian Jiu-Jitsu players. Arch Budo. 2012;8:103-7. 
Efeitos do equilíbrio em praticantes de Brazilian Jiu-Jitsu

ENDEREÇO

Bruno Brasil

Escola Superior de Educação Física Universidade Federal de Pelotas

R. Luiz de Camões, 625

Recebido para publicação: 12/ 12/ 2014

Revisado: $13 / 05 / 2015$

Aceito: 03/ 08/2015

96055-630 - Pelotas - RS - BRASIL

e-mail: brasil.costa@hotmail.com

Rev Bras Educ Fís Esporte, (São Paulo) 2015 Out-Dez; 29(4):535-41 • 541 